\title{
Detecting conjunctions of color and form in parallel
}

\author{
J. TOBY MORDKOFF, STEVEN YANTIS, and HOWARD E. EGETH \\ Johns Hopkins University, Baltimore, Maryland
}

\begin{abstract}
Certain theories of visual attention assume that at least one processing stage must be serial when the target of search is defined as the conjunction of two or more separable features. To explain why conjunction-search response times do not always form linearly increasing functions of display size, recent versions of this general model have posited the existence of an early parallel process that guides the serial stage toward display elements that are likely targets. Other models have relaxed the seriality assumption, allowing for a limited number of parallel decisions. In the three experiments reported here, a redundant-target detection task was used with conjunctively defined targets and display sizes of two (Experiment 1), one or two (Experiment 2), and six (Experiment 3). In all three experiments, strong evidence for parallel processing was observed. The implications for models of elementary visual processes are discussed.
\end{abstract}

Experiments in visual search provide an important class of tools for exploring the processes subserving early vision. For example, the analysis of response time as a function of display size has furnished significant evidence concerning the properties of the rate-limiting process underlying visual search (Egeth, Jonides, \& Wall, 1972; Treisman \& Gelade, 1980). Two qualitatively different patterns of results are typically observed when the number of display elements is varied from trial to trial. Which pattern is found depends on the nature of the target and nontarget stimuli. When search involves a target that differs from nontargets in a single salient feature (e.g., a vertical line among horizontal lines), then the searchtime function remains essentially flat over wide ranges of display size, supporting the notion that targets are detected by a spatially parallel process. In contrast, when the target is defined by a conjunction of features, each of which is separately present among the nontarget elements (e.g., a red X among red Os and black Xs), then search times increase linearly with display size, suggesting a serial, item-by-item search. This fundamental differ-

This research was supported by grants from NASA (JRI NCA2-413) and NIMH (R01-MH43924) to Steven Yantis and by AFOSR (87-0180) to Howard Egeth. Preliminary reports were made at the 60th Annual Meeting of the Eastern Psychological Association and the 1st Annual Meeting of the American Psychological Society. Some results from Experiments 1 and 2 will also appear in J. Pomerantz and G. Lockhead, (Eds.), The Perception of Structure (Washington, DC: The American Psychological Association). Katrina Van Valen, Robert Marra, Vibha Thakral, and Craig McDowell provided excellent technical assistance. We gratefully acknowledge the advice of William Bacon, Dale Dagenbach, Douglas Johnson, and Fred Merkel, as well as the comments of Charles W. Eriksen, Anne Treisman (who suggested the ancillary search experiment), and two anonymous reviewers. Correspondence should be sent to the authors at the Department of Psychology, The Johns Hopkins University, Baltimore, MD 21218. ence in performance has been the focus of much research over the past two decades (for reviews, see Cave \& Wolfe, 1990; Pashler, 1987; Treisman \& Gormican, 1988).

Recently, the evidence from a variety of visual search experiments has led theorists to propose models that together fall into a class known as two-stage models (Cave \& Wolfe, 1990; Duncan \& Humphreys, 1989; Hoffman, 1978, 1979; Sagi \& Julesz, 1985; Schneider \& Shiffrin, 1977; Shibuya \& Bundesen, 1988; Shiffrin \& Schneider, 1977; Treisman \& Sato, in press). According to these models, search is carried out in two sequential steps. In the first step, a rapid, somewhat error-prone, parallel analysis of the display is performed. The stimuli are ordered or prioritized according to various criteria (e.g., the number of features held in common with the target), but a final decision about target presence cannot be made. Only in the second stage can decisions be made and an overt response activated. Thus, the parallel stage acts as a guide for the second stage, pointing out likely targets or suppressing those display elements that are very dissimilar to the target.

One way to distinguish between specific two-stage models is to consider whether they allow more than one element to be processed simultaneously by the second stage. Some models assume that the second stage is strictly serial and that decisions about display elements are made one by one, in the order specified by the first stage (e.g., guided search; see Cave \& Wolfe, 1990; Hoffman, 1978, 1979; Sagi \& Julesz, 1985; Treisman \& Sato, in press). Others allow for a limited number of elements (usually about four) to be decided about in parallel (e.g., Duncan \& Humphreys, 1989; Fisher, 1982). Members of both model classes can account for the canonical data of feature integration theory (Treisman \& Gelade, 1980) as well as for some apparently contradictory results (e.g., Egeth, Virzi, \& Garbart, 1984; Wolfe, Cave, \& Franzel, 1989). The purpose of the present study was to collect evidence 
that would discriminate between these two classes of models.

\section{Testing for Spatially Parallel Processing}

The assumption that search for a conjunction of features requires serial processing (Cave \& Wolfe, 1990; Treisman \& Gelade, 1980) was recently questioned by Pashler (1987). He demonstrated that for displays of eight or fewer items, conjunction search appears to be carried out by a limited-capacity parallel process. In a series of experiments, the display-size functions for the targetabsent and target-present conditions were always increasing within this range, but they also had very similar slopes. Such a result contradicts serial self-terminating models, which predict a 2:1 ratio between the slopes of these two functions.

Pashler (1987) did not conclude that the observed 1:1 slope ratios indicated serial exhaustive processing (e.g., Sternberg, 1969), because this result is also consistent with certain parallel models (see Townsend, 1990; Townsend \& Ashby, 1983). To distinguish between these alternatives, Pashler conducted an experiment in which some displays contained two identical targets. Response times were faster with two targets than with only one. This redundancy gain is not consistent with a serial exhaustive model.

These two findings - a 1:1 slope ratio for small displays and a redundancy gain for redundant-target trialsconverge to raise questions about the seriality assumption of both feature integration theory and some more recent two-stage models, such as guided search. However, Pashler's (1987) argument for parallel decision processing is somewhat indirect in that it depends on the coordinated results from several experiments. The finding of a 1:1 slope ratio is inconsistent with serial self-terminating models, but it does not rule out serial exhaustive models; the finding of a redundancy gain is inconsistent with serial exhaustive models, but it does not rule out serial selfterminating models. Thus, the evidence for parallel processing exists only in the contradiction of both types of serial model.

A more direct test for parallel processing is the focus of the experiments reported here. We employed a redundant-target detection task and used a very conservative test for spatially parallel processing. Rather than manipulate the number of nontargets, as in visual search experiments, we manipulated the number of targets from trial to trial. Target-absent displays included no targets; target-present displays included either one or two targets.

A variety of models predict that mean response time should be faster when there are two targets present than when there is only one, and not all of these models involve parallel processing (e.g., Holmgren, Juola, \& Atkinson, 1974; van der Heijden, 1975). This is why Pashler's finding of a redundancy gain did not by itself rule out serial models. However, there is a specific form of redundancy gain that is inconsistent with any model that posits a strictly serial decision stage. Testing for this out- come requires an analysis of the entire distribution of response times.

Miller (1982) demonstrated that all models which assume that each target produces an independent, separate activation must satisfy the following inequality, called the race-model inequality:

$P\left(\mathrm{RT}<t \mid \mathrm{T}^{(1)} \& \mathrm{~T}^{(2)}\right) \leq P\left(\mathrm{RT}<t \mid \mathrm{T}^{(1)}\right)+P\left(\mathrm{RT}<t \mid \mathrm{T}^{(2)}\right)$,

where RT is response time, $t$ is time since stimulus onset, and $\mathrm{T}$ is a target in the superscripted spatial location. Thus, for example, $P\left(\mathrm{RT}<t \mid \mathrm{T}^{(1)} \& \mathrm{~T}^{(2)}\right)$ is the cumulative distribution function (CDF) for redundant targets, defined as the cumulative probability that a response has $o c-$ curred before time $t$, given that there is a target in both display locations.

One requirement of this inequality is that the fastest response times to displays with redundant targets be no faster than the fastest response times to single-target displays (although fast trials may happen more often with redundant targets). This is clearly the case for any serial model. When two targets are present, there is a higher probability that a target will be the first display element to be processed, but the fastest possible response will (in all conditions) occur when the target is processed first. In general, serial models are inconsistent with any violations of Inequality 1.

More importantly, the favored position models that have been presented as possible serial explanations of a redundancy gain (e.g., Biederman \& Checkosky, 1970; van der Heijden, La Heij, \& Boer, 1983; discussed in Mullin, Egeth, \& Mordkoff, 1988) cannot produce violations of Inequality 1 either. Thus, violations of this inequality constitute strong evidence against serial processing. ${ }^{1}$

\section{Overview}

The present study consists of three redundant-target detection experiments. The first two involved only small displays, one or two letters presented above and/or below fixation. These experiments formed a direct test of strictly serial, two-stage models of conjunction search (e.g., Cave $\&$ Wolfe, 1990). The third experiment was designed to test whether a slightly modified version of the two-stage serial model could explain the results of Experiments 1 and 2 . This required somewhat larger displays.

\section{EXPERIMENT 1}

In Experiment 1, we used the redundant-target detection task to examine whether conjunction search necessarily involves a serial stage. The number of targets was varied across trials from zero to one or two, and the response times to target-present displays were analyzed for violations of Inequality 1 . To ensure that displays were in fact examined for the presence of the conjunction target, rather than the mere presence of both of the target's 
features, a number of target-absent trials included both target features separately.

\section{Method}

Subjects. Twelve undergraduates at the Johns Hopkins University served as paid subjects. All had normal or corrected-to-normal vision. Each was paid about $\$ 5$ (depending on accuracy and speed; see below) for participating in a single 50-min session. None had participated in any similar experiment previously.

Apparatus and Stimuli. The stimuli were presented on a Princeton Graphics SR-12 RGB color monitor controlled by an IBM AT microcomputer equipped with a Sigma Design Color-400 EGA board. Each display consisted of two colored letters against a black background. The letters were positioned with their centers $1.2 \mathrm{~cm}$ above or below fixation. The letters were arranged vertically to minimize any effects of spatial stimulus-response compatibility (for evidence concerning the importance of this factor, see Grice, Canham, \& Boroughs, 1984). Each letter was $1.1 \mathrm{~cm}$ tall and $0.7 \mathrm{~cm}$ wide. From a viewing distance of $45 \mathrm{~cm}$, the eccentricity of the display locations from fixation was $1.53^{\circ}$, and the letters subtended $1.40^{\circ} \times 0.89^{\circ}$. The ratio between the height of the letters and their eccentricity from fixation was $1: 1.09$. The fixation cross was 0.5 $\times 0.5 \mathrm{~cm}\left(0.64^{\circ} \times 0.64^{\circ}\right)$. The subjects responded by pressing a button on a custom response box with the index finger of their dominant hands.

Design. For all subjects, the target was a red $X$ and the nontargets were red $O$ and green $X$. In each of the 10 blocks of 48 trials, half of the trials included at least one red $X$, and the other half included no targets. There were five different types of trials: (1) target in upper position, nontarget in lower; (2) target in lower position, nontarget in upper; (3) targets in both positions; (4) two different nontargets; and (5) two identical nontargets. Table 1 shows the frequency of occurrence for each type of trial. The order of trials was randomized prior to each block.

In this design, target presence is uncorrelated with whether the stimuli are the same or different: One third of all target-present trials had two identical elements, and one third of all target-absent trials had two identical elements. Furthermore, the inclusion of two different nontargets (one red $\mathrm{O}$ and one green $\mathrm{X}$ ) on two thirds of the target-absent trials forced subjects to search for the conjunction red $\mathrm{X}$, rather than the simple presence of both red and $\mathrm{X}$.
Procedure. Subjects were told that the target was the conjunction red $X$, that they should respond only on those trials that included at least one red $X$, and that they should refrain from responding on all other trials. A disjunctive-response or go/no-go paradigm was employed to reduce the possibility of response-competition effects (B. A. Eriksen \& C. W. Eriksen, 1974) on single-target trials (for a discussion of this issue, see van der Heijden, Schreuder, Maris, \& Neerincx, 1984). The subjects were also instructed that targets would appear on half of all trials, and that the nontargets were red Os and green Xs. Finally, the subjects were told that they would be rewarded (monetarily) for being fast and accurate.

Each trial began with the presentation of a dim white fixation cross at the center of the screen. After $350 \mathrm{msec}$, fixation was extinguished and the test stimuli appeared and remained visible until either a response was made or $1,000 \mathrm{msec}$ had elapsed. The following trial began after an intertrial interval of $2 \mathrm{sec}$.

A $700-\mathrm{Hz}$ tone sounded for $200 \mathrm{msec}$ following both miss and false alarm errors. Three such tones were sounded if the subject responded before the test stimulus appeared (i.e., when an "anticipation" response was made). Performance feedback was provided at the end of each block; this included accuracy (in percent), mean response time, and the number of bonus points earned. Bonus points were awarded as follows: 15 points for correctly not responding on target-absent trials, approximately 35 points (depending on RT) for responding on target-present trials, and a loss of 350 points for any error. ${ }^{2}$

Blocks were approximately 55 trials long, depending on the number of errors made by the subject. A block in which the subject made no errors was 53 trials long ( 5 warm-up trials plus 48 testing trials). Each error added 2 trials: a recovery trial, plus the rerunning of the error trial at some random point later in the block. A session consisted of a short (20-trial) practice block, followed by 10 testing blocks, of which only the last 8 were retained for analysis. The first 5 trials in each block, as well as any trial immediately following an error (i.e., recovery trials), were excluded from analysis.

\section{Results and Discussion}

Mean response time for the redundant-target condition was $303 \mathrm{msec}$. For the single-target condition, it was $332 \mathrm{msec}$. The observed redundancy gain of $29 \mathrm{msec}$ was

Table 1

Trial Frequencies per Block of 48 Trials: Experiments 1 and 2

\begin{tabular}{lllcc}
\hline \multicolumn{1}{c}{ Condition } & $\begin{array}{c}\text { Upper Display } \\
\text { Element }\end{array}$ & $\begin{array}{c}\text { Lower Display } \\
\text { Element }\end{array}$ & \multicolumn{2}{c}{ Frequency of Occurrence } \\
\hline Single target & Red X & Green X & 4 & Experiment 1 \\
with distractor & Red X & Red O & 4 & 2 \\
& Green X & Red X & 4 & 2 \\
Red O & Red X & 4 & 2 \\
Single target & Red X & (empty) & 0 & 4 \\
alone & (empty) & Red X & 0 & 4 \\
Redundant targets & Red X & Red X & 8 & 8 \\
Two different & Green X & Red O & 8 & 4 \\
nontargets & Red O & Green X & 8 & 4 \\
One nontarget & Green X & (empty) & 0 & 2 \\
alone & Red O & (empty) & 0 & 2 \\
& (empty) & Green X & 0 & 2 \\
Two identical & (empty) & Red O & 0 & 2 \\
nontargets & Green X & Green X & 4 & 4 \\
\hline
\end{tabular}


Table 2

Error Rates (in Percent): Experiments 1 and 2

\begin{tabular}{|c|c|c|}
\hline Condition & Experiment 1 & Experiment 2 \\
\hline \multicolumn{3}{|c|}{ Go Trials (Miss Errors) } \\
\hline Single target with distractor & 0.0 & 0.0 \\
\hline Single target alone & $\mathrm{n} / \mathrm{a}$ & 0.1 \\
\hline Redundant targets & 0.0 & 0.0 \\
\hline \multicolumn{3}{|c|}{ No-Go Trials (False Alarms) } \\
\hline Two different nontargets & 2.4 & 3.1 \\
\hline One nontarget alone & $\mathrm{n} / \mathrm{a}$ & 0.1 \\
\hline Two identical nontargets & 0.9 & 0.6 \\
\hline
\end{tabular}

significant $[t(11)=9.38, \mathrm{p}<.001]$. However, because single-target displays included a nontarget in the second display location, this result cannot be used for discriminating between parallel and serial models. There are exemplars within both model classes that can predict such a result. For example, the simplest (unguided) serial model predicts a redundancy gain under these conditions, because a target will be the first item to be evaluated on all redundant-target trials, but on half of the single-target trials, the target will be processed second. The redundancy gain only rules out the serial exhaustive model. Error data appear in Table 2.

The response-time data were then analyzed for violations of Inequality 1 . This analysis involved several steps. First, each subject's data were divided into two subsets: one for Blocks 3-6 and another for Blocks 7-10. This was done to reduce the influence of practice effects on the overall shape of the final group distributions.

In the second step, we applied the distribution-function correction procedure of Grice, Nullmeyer, and Spiker (1977), under which "twins" of each false alarm RT are removed from the correct-response distributions (see
C. W. Eriksen, 1988, for rationale). This was done separately for each of the two subsets of data from each subject. This procedure eliminates the contaminating effect of including fast-guess responses in a response-time distribution. $^{3}$

Next, we calculated two cumulative distribution functions for each subset of data. The first, which corresponds to the left side of Inequality 1 , is the CDF for the redundant-target condition. The second, corresponding to the right side of Inequality 1 , is the sum of the two singletarget CDFs (i.e., target in the upper location with a nontarget in the lower, and target in the lower location with a nontarget in the upper). Specific values for all CDFs were calculated at 19 points (quantiles), corresponding to the 5th through 95th percentiles at $5 \%$ intervals. When necessary, linear interpolation was used. Because this experiment included 12 subjects, these two steps were repeated 24 times ( 2 subsets $\times 12$ subjects). The two subsets for each subject were then combined by computing the mean RT at each quantile, yielding a pair of functions corresponding to the right and left sides of Inequality 1 .

To produce a group CDF for the redundant-target condition, the mean response time across subjects was obtained at each of the 19 quantiles. The same procedure was used to create the group CDF for the sum of the single-target conditions. This method, known as Vincentizing (Vincent, 1912), creates group distributions that retain the general shape of all constituent distributions (Ratcliff, 1979; Thomas \& Ross, 1980).

Figure 1 shows the corrected, group CDF for the redundant-target condition and the sum of the functions for the two single-target conditions. Note that from the 5 th to the 50th percentile, the distribution function for the redundant-target condition lies above and to the left of

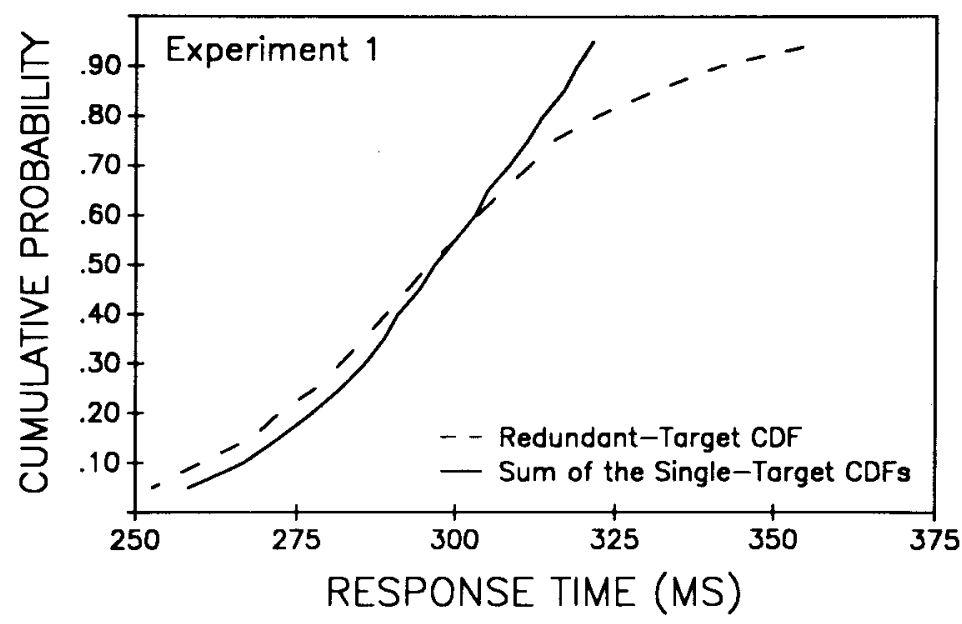

Figure 1. Group cumulative distribution functions from Experiment 1. (The solid line represents the sum of the functions from the two single-target conditions, and the dashed line represents the redundant-target condition.) 
the function representing the sum of the two single-target conditions. This constitutes a set of violations of Inequality 1 .

To test the significance of the observed violations, paired directional $t$ tests were conducted across subjects at each of the quantiles that showed a mean violation. The results of these tests revealed that Inequality 1 was significantly violated at the six quantiles corresponding to the 5th through 30th percentiles (all $p s<.05$, one-tailed). Table 3 summarizes these results.

Responses to redundant-target displays were too rapid to be consistent with any model that posits independent, separate activations. This includes the entire class of models positing a spatially serial decision process. Fast responses were not only more frequent on redundanttarget trials (a result that is consistent with certain serial models), but they were also faster than the fastest responses on single-target trials (a result that is not consistent with any serial model, nor with many parallel models; see Miller, 1982).

In considering the implications of Experiment 1, it is important to underscore how our results differ from those of previous work. Using an entirely different analytic tool for assessing whether processing is parallel or serialthe display-size diagnostic-many investigators have found that search for a conjunctively defined target produces a steep, linear function when reaction time is plotted against display size (e.g., Treisman \& Gelade, 1980). More recently, some investigators have found that, under certain conditions, reaction time in a conjunctionsearch task may increase only slightly, if at all, with display size (e.g., Nakayama \& Silverman, 1986; Wolfe et al., 1989). This is one sort of evidence for a parallel stage in processing. It is not yet clear, however, what stimulus conditions lead to steep or flat search functions; nor can these data establish whether the flat functions, when observed, are due to spatially parallel decision processing or just more efficient selection by means of some predecisional mechanism (cf. Pashler \& Badgio, 1985).

Of interest, therefore, is whether the stimuli used in the present experiments are of the type that lead to steep display-size functions. We examined this issue in an ancillary visual search experiment with display sizes 4, 9, 16, and 25. The task consisted of go/no-go target detection, and both display density and target eccentricity were controlled in the manner used previously by Treisman (e.g., Treisman \& Sato, in press). Under these conditions, target-present response time increased by 26 msec per item $(p<.01)$ and the function was $91 \%$ linear. Thus, our evidence for parallel processing should not be considered a special case involving an especially easy target-distractor discrimination. That stimuli which produce steep response-time functions in search tasks can nonetheless also result in violations of Inequality 1 demonstrates another possible limitation to the display-size diagnostic (cf. Townsend, 1990).

\section{EXPERIMENT 2}

An alternative account of any violation of Inequality 1 holds that responses are not extra fast when there are redundant targets, but that they are slowed on single-target trials due to the presence of a nontarget in the second display location. In other words, it is possible that redundancy gains are not actually due to a gain on redundanttarget trials, but that they are instead associated with a loss on single-target trials (for discussions, see C. W. Eriksen, Goettl, St. James, \& Fournier, 1989; Grice et al., 1984; van der Heijden et al., 1984). According to this view, a distraction decrement caused by the nontarget included in single-target displays yields the observed

Table 3

Violations of Inequality 1: Experiment 1

\begin{tabular}{|c|c|c|c|c|}
\hline Percentile & $\begin{array}{c}\text { Redundant-Target } \\
\text { Distribution }\end{array}$ & $\begin{array}{c}\text { Sum Single-Target } \\
\text { Distribution }\end{array}$ & $\begin{array}{l}\text { Violation of } \\
\text { Inequality } 1\end{array}$ & $t(11)$ \\
\hline 05 & 253 & 258 & 5 & $1.97^{*}$ \\
\hline 10 & 260 & 267 & 7 & $3.06^{*}$ \\
\hline 15 & 268 & 272 & 4 & $2.73 *$ \\
\hline 20 & 272 & 277 & 5 & $3.28^{*}$ \\
\hline 25 & 278 & 282 & 4 & $2.11^{*}$ \\
\hline 30 & 282 & 286 & 4 & $2.01^{*}$ \\
\hline 35 & 286 & 289 & 3 & 1.42 \\
\hline 40 & 289 & 291 & 2 & 0.84 \\
\hline 45 & 293 & 294 & 1 & 0.93 \\
\hline 50 & 296 & 297 & 1 & 0.37 \\
\hline 55 & 300 & 300 & & \\
\hline 60 & 304 & 303 & & \\
\hline 65 & 308 & 305 & & \\
\hline 70 & 312 & 309 & & \\
\hline 75 & 315 & 311 & & \\
\hline 80 & 322 & 314 & & \\
\hline 85 & 331 & 317 & & \\
\hline 90 & 342 & 319 & & \\
\hline 95 & 358 & 322 & & \\
\hline
\end{tabular}


violations of Inequality 1 . This account is supported by the fact that, in previous research, violations have usually been observed only when single-target trials included nontargets; when single-target displays consisted of only a target (with the other display location left empty), violations of Inequality 1 have seldom been found (e.g., Grice et al., 1984; Miller, 1982; van der Heijden et al., 1984).

In Experiment 1, display size was fixed at two. Thus, redundant-target displays only contained targets, but single-target displays always included a nontarget distractor. This leaves the results open to counterinterpretation through reference to a distraction decrement. Experiment 2 was designed to test this alternative by including a number of trial types with empty nontarget locations. There were now two types of single-target trials: single target with distractor and single target alone. If the results of Experiment 1 were due to a distraction decrement, then no violations of Inequality 1 should be observed when responses from the single-target-alone condition are compared with those from the redundant-target condition.

\section{Method}

The apparatus, stimuli, and procedure were the same as for Experiment 1 , except that four additional types of trials were added. These new trials all involved leaving one of the two display locations empty. There were nine different types of trials: (1) target in upper position, nontarget in lower; (2) target in lower position, nontarget in upper; (3) target in upper position, lower position left empty; (4) target in lower position, upper position empty; (5) targets in both positions; (6) two different nontargets; (7) nontarget in upper position, lower position empty; (8) nontarget in lower po-

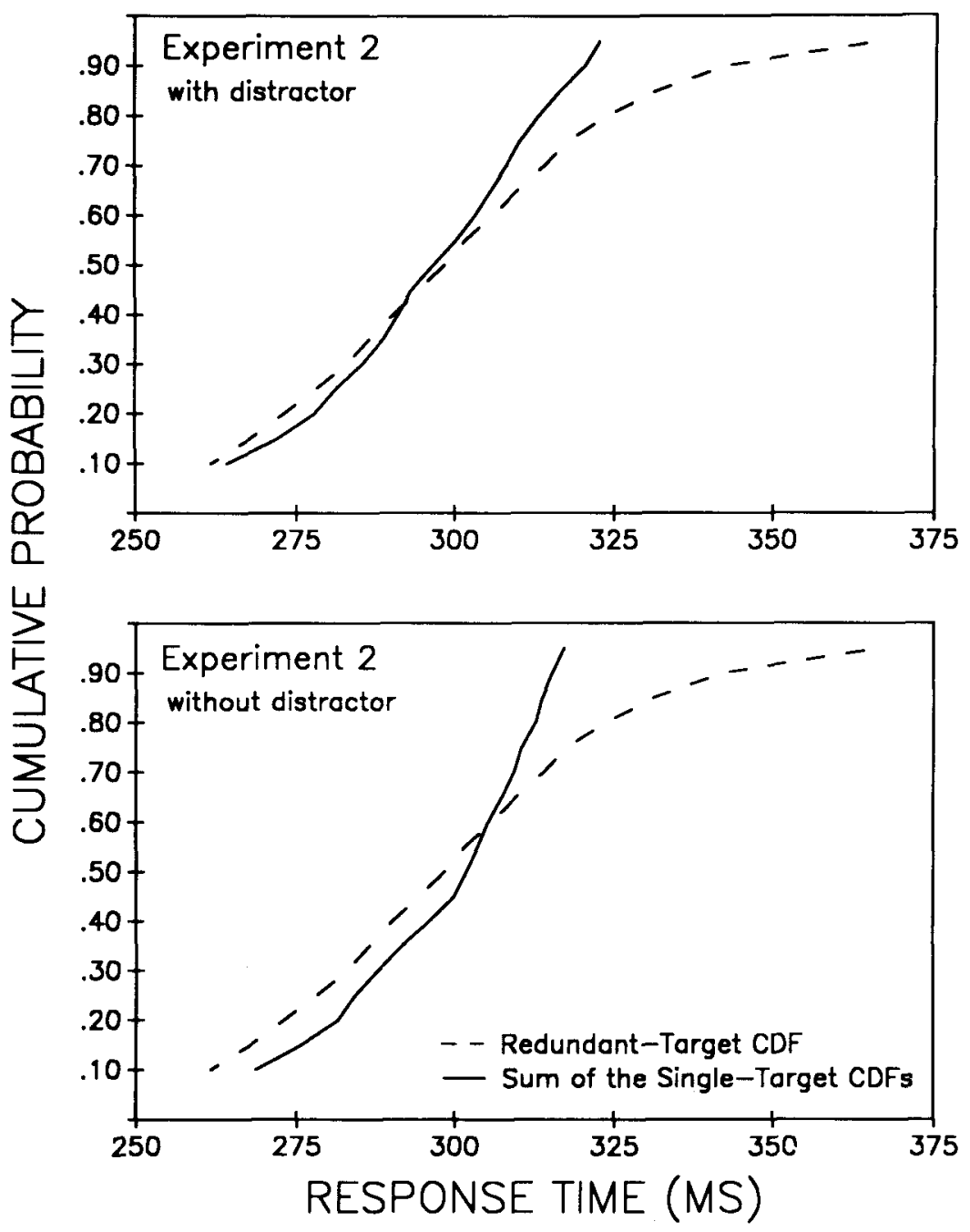

Figure 2. Group cumulative distribution functions from Experiment 2. (The upper panel compares the redundant-target condition to the single-target-with-distractor condition. The lower panel compares the redundant-target condition to the single-target-alone condition. In each, the solid line represents the sum of the single-target conditions, and the dashed line represents the redundant-target condition.) 
sition, upper position empty; and (9) two identical nontargets. Table 1 lists the frequency of each type of trial.

As was true for Experiment 1, whether or not a response should be made was uncorrelated with whether the stimuli on a trial were same or different. Display size was not a source of useful information either: Half of all one-element displays included a target; half did not. Twelve new subjects were recruited from the same pool as in Experiment 1 . As before, subjects were given a short (20trial) practice block, followed by 10 testing blocks of about 55 trials; only the last 8 full blocks were retained for analysis.

\section{Results and Discussion}

Mean response times for redundant targets, single target with distractor, and single target alone were 305, 337, and $328 \mathrm{msec}$, respectively. The redundancy gain was significantly greater than zero, regardless of whether or not a distractor was included in single-target displays. The difference between the means for single target with distractor and redundant targets was $32 \mathrm{msec}[r(11)=11.37$, $p<.001]$. The difference between single target alone and redundant targets was $23 \mathrm{msec}[t(11)=8.46, p<$ .0011 . Finally, the 9 -msec difference between single target with distractor and single target alone was also significant $[t(11)=2.73, p<.025]$. The error data appear in Table 2.

For this experiment, two separate tests of Inequality 1 were conducted: one comparing redundant targets to single target with distractor (Figure 2, upper panel); another comparing redundant targets to single target alone (Figure 2, lower panel). As in Experiment 1, the data from a given subject were divided into two subsets, and "twins" of false alarm responses were removed from the data before calculating the individual or group CDFs. Note that the first quantile for these analyses was placed at the 10th percentile. The 5th percentile was never cal- culated, because there were sometimes too few observations after "twin-killing" to do so without extrapolation.

In both sets of $t$ tests, significant violations of Inequality 1 were observed. In the analysis involving the singletarget-with-distractor condition, violations were observed from the 10th through 40th percentiles. The violations at the 15th and 20th percentiles were significant at the .05 level (one-tailed). For the analysis using the single-targetalone condition, Inequality 1 was violated at the 10th through 55th percentiles, with the violations at the 10th, 15 th, and 20 th percentiles being significant. Table 4 summarizes these results.

These consistent violations of Inequality 1 provide evidence against models that incorporate a strictly serial stage prior to response selection. That this result was obtained even when single-target displays did not include a nontarget rules out the alternative account that violations are due to a distraction decrement. The small but significant effect of including a nontarget could alternatively be due to a limit in perceptual capacity, to mutual interference (masking) in the processing of two-element displays, or to a small proportion of strictly serial trials.

\section{EXPERIMENT 3}

Although Experiments 1 and 2 provide strong evidence contradicting strictly serial, two-stage models such as guided search (Cave \& Wolfe, 1990), one might ask whether there are still other (more complex) models incorporating a serial decision stage that could explain the observed violations of Inequality 1 . One such model would retain the notion of strictly serial decisions, but to this would be added the notion that other processes are simultaneously at work during target search. Rather than

Table 4

Violations of Inequality 1: Experiment 2

\begin{tabular}{|c|c|c|c|c|c|}
\hline Percentile & $\begin{array}{c}\text { Redundant-Target } \\
\text { Distribution }\end{array}$ & $\begin{array}{l}\text { Sum Single-Target- } \\
\text { with-Distractor } \\
\text { Distribution }\end{array}$ & $\begin{array}{c}\text { Violation for } \\
\text { Single Target } \\
\text { with Distractor }\end{array}$ & $\begin{array}{l}\text { Sum Single-Target- } \\
\text { Alone Distribution }\end{array}$ & $\begin{array}{c}\text { Violation for } \\
\text { Single Target Alone }\end{array}$ \\
\hline 10 & 262 & 264 & 2 & 269 & 7* \\
\hline 15 & 268 & 272 & $4^{*}$ & 276 & $8^{*}$ \\
\hline 20 & 273 & 278 & $5^{*}$ & 282 & 9* \\
\hline 25 & 278 & 281 & 3 & 284 & 6 \\
\hline 30 & 283 & 286 & 3 & 288 & 5 \\
\hline 35 & 287 & 289 & 2 & 292 & 5 \\
\hline 40 & 290 & 291 & 1 & 296 & 6 \\
\hline 45 & 294 & 293 & & 300 & 6 \\
\hline 50 & 298 & 297 & & 302 & 4 \\
\hline 55 & 302 & 300 & & 304 & 2 \\
\hline 60 & 306 & 303 & & 305 & \\
\hline 65 & 310 & 306 & & 308 & \\
\hline 70 & 314 & 308 & & 309 & \\
\hline 75 & 318 & 310 & & 311 & \\
\hline 80 & 324 & 313 & & 313 & \\
\hline 85 & 332 & 316 & & 314 & \\
\hline 90 & 343 & 320 & & 315 & \\
\hline 95 & 367 & 322 & & 317 & \\
\hline
\end{tabular}

Note-Statistical tests are one-tailed. Tabled values are in milliseconds. ${ }^{*} p<.05$. 
being only a two-stage model, this alternative is a twostage, two-process model. This model involves no spatially parallel decision processes, but allows for functionally parallel processes to contribute simultaneously to response activation.

The central process in this hybrid model would perform a guided search for targets. To be consistent with assertions that conjunction search is necessarily serial, we will assume that the display elements would therefore be worked through one at a time. By means of the other perceptual process, the two maps associated with the target's two features would be monitored (Treisman, 1985), and any activation found would be passed to the mechanisms that select a positive response. In order to avoid causing false alarms on trials that include both target features but no targets, this second process would never produce enough activation to trigger a response by itself. Thus, although the rate-limiting, final-decision process of this hybrid model would be serial, the secondary, mapmonitoring process could still produce enough activation to speed responding on trials that include multiple target features.

To see how the two-stage, two-process model could yield violations of Inequality 1 without actually processing more than one conjunction simultaneously, consider the following: In Experiments 1 and 2, all displays that contained redundant targets also included more target features than did the displays that contained only one target (four features on redundant-target trials as opposed to only two or three on single-target trials). If the amount of activation produced by the map-monitoring process is in any way dependent on the number of target features present, it is possible that this second process-which never actually conjoins features-was responsible for the extra-fast responses on redundant-target trials in Experiments 1 and 2.

Although this particular model may seem implausible, it was necessary to test it before firmly concluding against all strictly serial two-stage models. ${ }^{4}$ We did so by holding the number of target features constant across trials while still manipulating target numerosity. This required that display size be increased to six.

\section{Method}

As in Experiments 1 and 2, the target was always the conjunction red $X$. To maintain a constant number of target features (i.e., redness and $X$ shapes), each display contained two red, two green, and two blue elements; they also contained two Xs, two Os, and two Is.

To keep the correlation between target presence and number of identical items at zero (i.e., to ensure that display homogeneity was not a source of useful information), all displays included exactly one pair of identical items. ${ }^{5}$ For example, a target-absent trial could consist of a red $O$, a red I, a green $O$, a green I, and two blue Xs. A single-target trial could contain a red $X$ (the target), a red $O$, a green $X$, a green $O$, and two blue Is. All redundant-target trials consisted of two red $X s$, a green $O$, a green $I$, a blue $O$, and a blue $I$.

All letters were $1.1 \mathrm{~cm}$ tall and $0.7 \mathrm{~cm}$ wide. From a viewing distance of $45 \mathrm{~cm}$, the letters subtended $1.40^{\circ} \times 0.89^{\circ}$ visual angle. Display locations were placed at equal intervals along the cir- cumference of an imaginary circle that had a radius of $1.5 \mathrm{~cm}$ $\left(1.91^{\circ}\right)$ and was centered at fixation. This yields an element-sizeto-eccentricity ratio of $1: 1.36$ (which is slightly lower than that used in Experiments 1 and 2). The location for a given element was selected at random on each trial. Thus, not all displays containing the same six objects were identical.

One sixth of all trials contained redundant targets. One third were single-target trials, and the remaining half contained no targets. As in Experiments 1 and 2, the probability that at least one target was present was maintained at 0.5 , and a go/no-go task was used. The amount of time that displays remained visible before a trial was considered a no-go was $1,000 \mathrm{msec}$.

Six new subjects were recruited, each participating at the same hour on 3 consecutive days. Sessions consisted of a short (20-trial) practice block and 10 blocks of testing. As before, the first two full blocks and the first 5 trials of each subsequent block (as well as all trials immediately following an error) were discarded.

\section{Results and Discussion}

For all three sessions, responses to redundant-target displays were faster than those to single-target displays. Redundancy gains in Sessions 1, 2, and 3, respectively, were 35,37 , and $37 \mathrm{msec}[t(5)=14.03,8.63$, and 11.16 , respectively, all $p \mathrm{~s}<.001]$. However, because display size was fixed at six, resulting in fewer nontargets on redundant-target trials as compared with single-target trials, this result does not allow us to discriminate serial from parallel models. Error data appear in Table 5.

In order to perform tests of Inequality 1, responses to all single-target trials were split randomly into two subsets. The CDFs from these subsets were then summed to form the right side of Inequality 1 . After the removal of false alarm "twins," three separate tests of Inequality 1 were conducted, one for each experimental session. Analysis of Session 1 revealed no violations of Inequality 1 . Session 2 included violations from the 5 th to 25 th percentiles (Figure 3, upper panel), with that at the 5th percentile being significant at the .05 level (one-tailed). Results from Session 3 violated the inequality from the 5th to 40th percentiles (Figure 3, lower panel), with those at the 5th, 10th, and 20th percentiles being statistically significant. Table 6 summarizes the results from Session 3.

Although not appearing until after some practice, violations of Inequality 1 reached significance in Experiment 3 . This result rules out the hybrid two-stage, twoprocess model described earlier. In considering these results beside those of Experiments 1 and 2, there are several interpretations of why additional experience with the task was required before violations appeared. For ex-

Table 5

Error Rates (in Percent): Experiment 3

\begin{tabular}{lccc}
\hline \multicolumn{1}{c}{ Condition } & Session 1 & Session 2 & Session 3 \\
\hline \multirow{4}{*}{ Single target } & Go Trials (Miss Errors) & \\
Redundant targets & 0.1 & 0.0 & 0.1 \\
& 0.0 & 0.0 & 0.3 \\
All & No-Go Trials (False Alarms) & \\
\hline
\end{tabular}




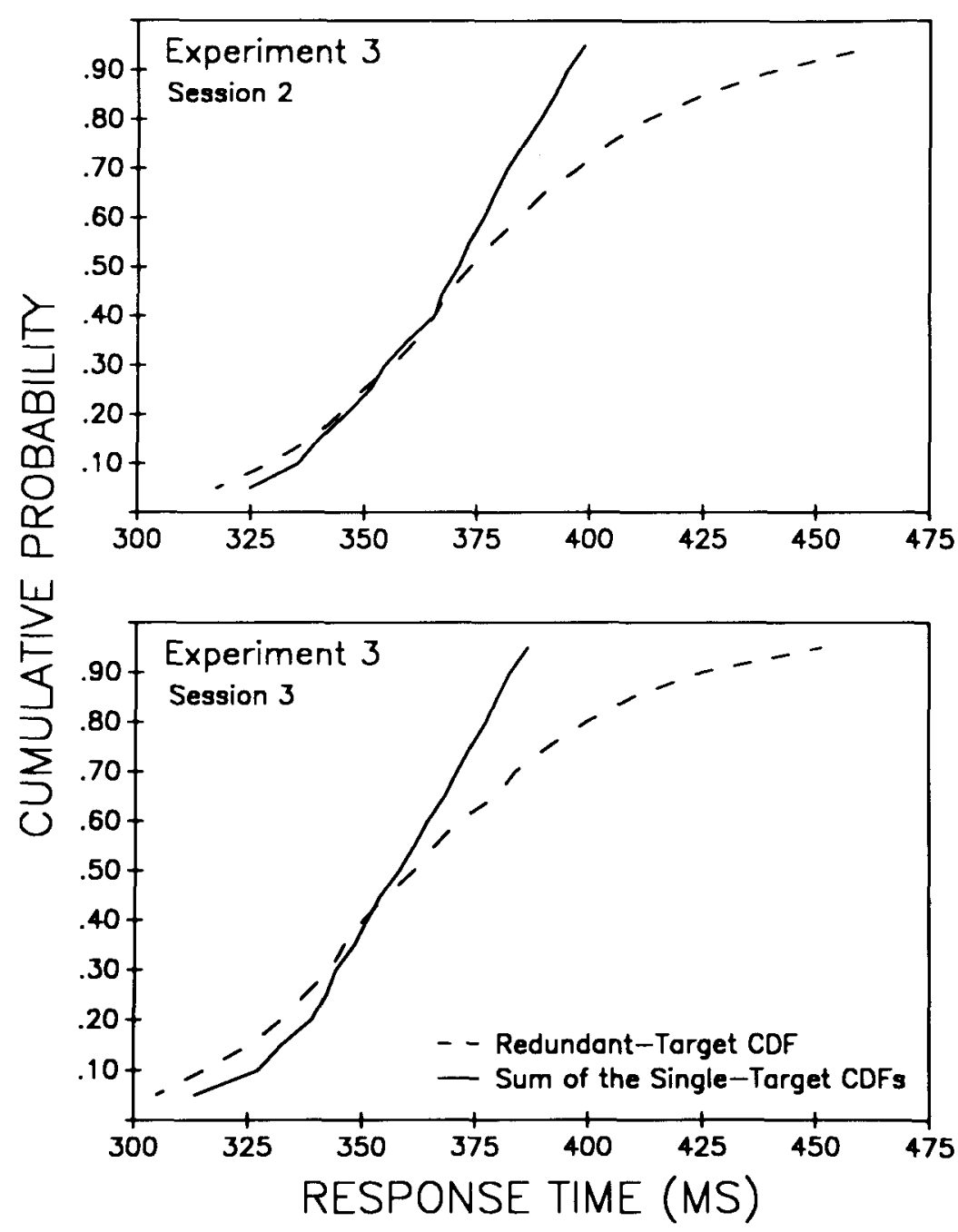

Figure 3. Group cumulative distribution functions from Experiment 3. (The upper panel is from Session 2, the lower from Session 3. In each, the solid line represents the sum of the single-target conditions, and the dashed line represents the redundant-target condition.)

ample, it may be that differences in the amount of perceptual capacity required by two-location and six-location experiments are such that performance efficiency changes more slowly in the latter situation. Alternatively, the difference could relate to the lower element-size-toeccentricity ratio used in Experiment 3 (Duncan \& Humphreys, 1989). Regardless of why added practice was necessary, however, the appearance of any violation of Inequality 1 rules out the two-process model targeted by this experiment (as well as the feature-map explanation suggested by Treisman, 1988; see also Note 4). Thus, the results of the three experiments taken together rule out the strict seriality assumption of certain models of conjunction search, including models that include a preattentive parallel stage that serves to guide a serial decision device toward targets (Cave \& Wolfe, 1990) or away from nontargets (Treisman \& Sato, in press).

\section{GENERAL DISCUSSION}

For nearly a decade, a great deal of attention research has focussed on feature integration theory (Treisman \& Gelade, 1980). One of the most attractive elements of this model is its simplicity. The two patterns of target-search data are each explained by reference to a different aspect of a single model. When the target of search is defined as a value along one feature dimension (e.g., an X among Os), search is display-size-independent because attention is not required. When the target is defined as a conjunction of two or more features, search is serial because 
Table 6

Violations of Inequality 1: Session 3 of Experiment 3

\begin{tabular}{cccc}
\hline Percentile & $\begin{array}{c}\text { Redundant-Target } \\
\text { Distribution }\end{array}$ & $\begin{array}{c}\text { Sum Single-Target } \\
\text { Distribution }\end{array}$ & $\begin{array}{c}\text { Violation of } \\
\text { Inequality I }\end{array}$ \\
\hline 05 & 305 & 313 & $8^{*}$ \\
10 & 315 & 327 & $12^{*}$ \\
15 & 326 & 333 & 7 \\
20 & 333 & 339 & $6^{*}$ \\
25 & 338 & 342 & 4 \\
30 & 343 & 344 & 1 \\
35 & 346 & 348 & 2 \\
40 & 350 & 351 & 1 \\
45 & 355 & 354 & \\
50 & 361 & 358 & \\
55 & 366 & 361 & \\
60 & 371 & 364 & \\
65 & 380 & 368 & \\
70 & 384 & 371 & \\
75 & 391 & 374 & \\
80 & 400 & 377 & \\
85 & 410 & 380 & \\
90 & 425 & 383 & \\
95 & 451 & 387 & \\
\hline
\end{tabular}

Note-Statistical tests are one-tailed. Tabled values are in milliseconds ${ }^{*} p<.05$.

attention is required to conjoin separable features into a unified percept that is compared with the target representation.

In light of the mounting evidence against the original form of feature integration theory (e.g., Egeth et al., 1984; Houck \& Hoffman, 1986; McLeod, Driver, \& Crisp, 1988; Nakayama \& Silverman, 1986; Pashler, 1987; Steinman, 1987; Wolfe et al., 1989), two classes of alternative models have been proposed. These two classes are most easily distinguished by whether their members allow for spatially parallel decision processing. Guided serial models (e.g., Cave \& Wolfe, 1990; Hoffman, 1978, 1979) assume strictly serial decision processing, whereas limited-capacity parallel models (e.g., Duncan \& Humphreys, 1989; Pashler, 1987) assume that a small number of display elements may be processed simultaneously.

\section{Two-Stage Guided Serial Models}

Guided serial models never allow decisions to be made about more than one display item at once. In fact, the model proposed by Cave and Wolfe (1990) requires serial processing even when the target is defined by a single feature. The qualitative differences in performance under feature-search and conjunction-search conditions are explained through reference to an error-prone or noisy parallel stage that guides the serial decision device. When targets include a conspicuous feature not included in any of the nontargets, the parallel stage makes few mistakes and the target is usually the first element to be scanned. When two or more dimensions are involved, as in search for a conjunction of features, more errors arise in the parallel stage and response time increases with the number of display elements. In this way, guided serial models provide a route around some of the demonstrated limitations of feature integration theory.
But however attractive this class of models may seemand the single-mechanism explanation provided by guided search is, at least, parsimonious-it is at odds with the results of the present study. With the redundant-target detection paradigm and application of Miller's (1982) racemodel inequality (Inequality 1), we have provided evidence that the assumption of strictly serial processing is violated. Under some circumstances, at least two display elements may affect decision at the same time. This conclusion may force modification of the strict seriality assumption of guided search. Experiment 3, however, has ruled out one alternative that retains the serial decision stage.

In defense of guided search (Cave \& Wolfe, 1990), one might counter that in the present experiments we employed only small display sizes with occasional redundant targets and that our results would apply only to those conditions. In other words, one could argue that there is some crucial theoretical difference between target-detection tasks (where displays are small) and visual search tasks (where displays are often quite large), and that different kinds of models are required for each. We would counter, however, that a single model capable of accounting for both conditions is preferable to separate models.

\section{Limited-Capacity Parallel Models}

As mentioned above, there are two classes of alternatives to feature integration theory. A central assumption of two-stage, guided serial models has been called into question by the present and previous evidence of true parallel processing. The second class of models must now be considered. These models do allow for spatially parallel processing of integrated objects (i.e., feature conjunctions), but they also assume that there is some limit to the capacity of the decision stage. For example, as the required discriminations become more difficult (e.g., as the number of features shared by targets and nontargets increases), the number of elements that may affect decision in parallel decreases.

Pashler (1987) suggested that conjunction search was a limited-capacity parallel process with a maximum of about eight simultaneously encoded stimuli. When displays contain more than eight items, subsets of about eight items are searched in series. In other words, small clumps of the display are searched in parallel, but global search (among clumps) is serial. Treisman and Souther (1985) suggested a similar mechanism for certain difficult conditions of feature search (see also, Treisman, 1988; Treisman \& Gormican, 1988; Treisman \& Sato, in press); Duncan and Humphreys (1989) have added a preattentive stage prior to decision that specifies the order in which items gain access to visual short-term memory (a limitedcapacity, parallel mechanism).

Admittedly, the present results provide no direct evidence in favor of the latter models, nor any way of discriminating among them, but they are at least consistent with the notion of a parallel decision stage. Limitedcapacity parallel models have the additional virtue of be- 
ing able to account for results from both small-display and large-display experiments. Further evidence concerning the properties of this class of models is now needed.

\section{REFERENCES}

Biederman, I., Checkosky, S. F. (1970). Processing redundant information. Journal of Experimental Psychology. 83, 486-490.

CAve, K. R., \& Wolfe, J. M. (1990). Modeling the role of parallel processing in visual search. Cognitive Psychology, 22, 225-27!.

DunCAN, J., Humphreys, G.W. (1989). Visual search and stimulus similarity. Psychological Review, 96, 433-458.

Egeth, H. E., Jonides, J., \& WALl, S. (1972). Parallel processing of multielement displays. Cognitive Psychology, 3, 674-698.

Egeth, H. E., Virzi, R., Garbart, H. (1984). Searching for conjunctively defined targets. Joumal of Experimental Psychology: Human Perception \& Performance, 10, 32-39.

ERIKSEN, B. A., ERIKSEN, C. W. (1974). Effects of noise letters upon the identification of a target letter in a nonsearch task. Perception \& Psychophysics. 16, 143-149.

ERIKSEN, C. W. (1988). A source of error in attempts to distinguish coactivation from separate activation in the perception of redundant targets. Perception \& Psychophysics, 44, 191-193.

Eriksen, C. W., Goettl, B., St. James, J. D. , Fournier, L. R (1989). Processing redundant signals: Coactivation, divided attention, or what? Perception \& Psychophysics, 45, 356-370.

FISHER, D. L. (1982). Limited-channel models of automatic detection Capacity and scanning in visual search. Psychological Review, 89. 662-692.

Grice, G. R., Canham, L., \& Boroughs, J. M. (1984). Combination rule for redundant information in reaction time tasks with divided attention. Perception \& Psychophysics, 35, 451-463.

Grice, G. R., Nullmeyer, R., \& Siker, V. A. (1977). Application of variable criterion theory to choice reaction time. Perception \& Psychophysics, 22, 431-449.

Hofrman, J. E. (1978). Search through a sequentially presented visual display. Perception \& Psychophysics, 23, 1-11.

Hoffman, J. E. (1979). A two-stage model of visual search. Perception \& Psychophysics, 25, 319-327.

Holmgren, J. E., Juola, J. F., Atkinson, R. C. (1974). Response latency in visual search with redundancy in the visual display. Perception \& Psychophysics, 16, 123-128.

Houck, M. R., \& Hoffman, J. E. (1986). Conjunction of color and form without attention: Evidence from an orientation-contingent color aftereffect. Journal of Experimental Psychology: Human Perception \& Performance, 12, 186-199.

McLeod, P., Driver, J., \& Crisp, J. (1988). Visual search for a conjunction of movement and form in parallel. Nature, 332, 154-155.

MilleR, J. (1982). Divided attention: Evidence for coactivation with redundant signals. Cognitive Psychology, 14, 247-279.

Mullin, P. A., Egeth, H. E., \& Mordkoff, J. T. (1988). Redundanttarget detection and processing capacity: The problem of positional preferences. Perception \& Psychophysics, 43, 607-610.

Nakayama, K., Silverman, G. H. (1986). Serial and parallel processing of visual feature conjunctions. Nature, 320, 264-265.

Pashler, H. (1987). Detecting conjunctions of color and form: Reassessing the serial search hypothesis. Perception \& Psychophysics, 41 , 191-201

Pashler, H., \& Badgio, P. C. (1985). Visual attention and stimulus identification. Journal of Experimental Psychology: Human Perception \& Performance, 11, 105-121.

RATCLIFF, R. (1979). Group reaction time distributions and an analysis of distribution statistics. Psychological Bulletin, 86, 446-461

SAGl, D., \&ULESZ, B. (1985). "Where" and "what" in vision. Science, 228, $1217-1219$.

SCHNEIDER, W, \& Shiffrin, R. M. (1977). Controlled and automatic human information processing: 1 . Detection, search, and attention. Psychological Review, 84, 1-66.

Shibuya, H., Bundesen, C. (1988). Visual selection from multiele- ment displays: Measuring and modeling effects of exposure duration. Journal of Experimental Psychology: Human Perception \& Performance, 14, 591600 .

Shiffrin, R. M. . SChneider. W. (1977). Controlled and automatic human information processing: Il. Perceptual learning, automatic attending, and a general theory. Psychological Review, 84, 127-190.

Steinman. S. B. (1987). Serial and parallel search in partem vision? Perception, 16, 389-398.

Sternaera, S. (1969). Memory scanning: Mental processes revealed by reaction-time experiments. American Scientist, 57, 421-457.

Thomas, E. A. C. \& Ross, B. H. (1980). On appropriate procedures for combining probability distributions within the same family. Joumal of Mathematical Psychology, 21, 136-152.

TOWNSEND, J. T. (1990). Serial vs. parallel processing: Sometimes they look like Tweedledum and Tweedledee but they can (and should) be distinguished. Psychological Science, 1, 46-54.

Townsend, J. T., AshBY, G. (1983). Stochastic modeling of elementary psychological processes. Cambridge, England: Cambridge University Press.

Treisman, A. (1982). Perceptual grouping and attention in visual search for features and for objects. Joumal of Experimental Psychology: $\mathrm{Hu}$ man Perception \& Performance, 8, 194-214.

Treisman, A. (1985). Preattentive processing in vision. Computer Vision. Graphics, \& Image Processing, 31, 156-177.

Treisman, A. (1988). Features and objects: The fourteenth Bartlett memorial lecture. Quarterly Joumal of Experimental Psychology, 40A, 201-237.

Treisman, A. \& Gelade, G. (1980). A feature integration theory of attention. Cognitive Psychology, 12, 97-136.

Treisman, A., Gormican, S. (1988). Feature analysis in early vision. Psychological Review, 95, 15-48.

Treisman, A., \& SATo, S. (in press). Conjunction search revisited. Journal of Experimental Psychology: Human Perception \& Performance.

Treisman, A. M. . Souther, J. (1985). Search asymmetry: A diagnostic for preattentive processing of separable features. Journal of Experimental Psychology: General, 114, 285-310.

van der Hejden, A. H. C. (1975). Some evidence for a limited capacity parallel selfterminating process in simple visual search tasks. Acta Psychologica, 39, 21-41.

van der Heuden, A. H. C., La Heis, W., \& Boer, J. P. A. (1983), Parallel processing of redundant targets in simple search tasks. Psychological Research, 45, 235-254.

van der Heuden, A. H. C., Schreuder, R., Maris, L., \& Neerincx, M. (1984). Some evidence for correlated separate activation in a simple letter-detection task. Perception \& Psychophysics, 36, 577-585.

VINCENT, S. B. (1912). The function of the viborissae in the behavior of the white rat. Behavioral Monographs, $1($ No. 5$)$.

Wolfe, J. M., Cave, K. R., \& Franzel, S. L. (1989). Guided search: An alternative to the feature integration model for visual search. Journal of Experimental Psychology: Human Perception \& Performance. 15. 419-433.

\section{NOTES}

1. Miller (1982) interpreted violations of Inequality 1 as evidence for a special form of parallel processing called coactivation. For the present purposes, however, we are concerned only that such violations rule out all strictly serial models.

2. The use of this payoff matrix was very successful in keeping accuracy levels extremely high. In all three experiments, no one condition ever contained more than $0.3 \%$ miss errors or $3.1 \%$ false alarms. Anticipations occurred on less than $0.1 \%$ of all trials. Overall accuracy rates were above $98 \%$ in all experiments. Furthermore, accuracy was always higher in redundant-target than in single-target conditions, ruling out a speed-accuracy tradeoff explanation of the observed redundancy gains. For these reasons, accuracy data (other than anticipations) are presented in Tables 2 and 5, but they will not be discussed.

3. Seperate analyses for all experiments were also conducted using uncorrected data. In each case, the results were qualitatively identical.

4. There was a second reason for considering this experiment: According to a close reading of feature integration theory (see especially 
Treisman, 1982, 1988), the conditions of Experiments 1 and 2 might not have been sufficient to force subjects to process each display element as a distinct object. This holds because, for example, the redundanttarget displays included only target features (red and X), so the only objects possible were targets (red Xs). Thus, according to Treisman, the conditions of Experiments 1 and 2 might not have been sufficient to test her theory. (We thank Anne Treisman for pointing this out.) However, the results from our first two experiments still contradict the seriality assumption of Cave and Wolfe's (1990) guided search model, and those from Experiment 3 pose some problems for feature integration theory as well.
5. It is this particular constraint that required a display size of six If the number of identical items is allowed to vary across conditions, then a display size of four can be used: Each display would contain two $\mathrm{Xs}$ and two red items, with the number of targets varying from zero to two. This experiment was actually conducted, and it produced results similar to those in Experiment 3. It is not reported, due to the possible confounding effect of varying display homogeneity.

(Manuscript received November 20, 1989; accepted for publication February 9, 1990.) 